\title{
Propuesta de modelo integral de evaluación sostenible de la vivienda social en México
}

\author{
Sustainable assessment model proposal for social housing \\ in México
}

\section{Salvador Garcia Rodriguez \\ Miguel Davis Campoy \\ Eva Campos Cantu \\ Elizabeth Leyva Orihuela}

\section{Resumen}

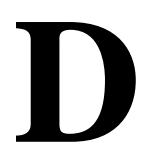

ebido al gran impacto de la industria de la construcción en el medio ambiente, la tendencia de la construcción sustentable a nivel mundial es una gran necesidad. La industria de la construcción es la principal actividad humana consumidora de recursos naturales por lo que se han creado herramientas que certifican el nivel de sustentabilidad e impacto ambiental de los edificios. En México aún no se ha desarrollado alguna metodología que evalue el grado de sustentabilidad de vivienda, por lo que se ha visto la necesidad de implementar modelos extranjeros, diseñados por otros países y para otros contextos, no viables de aplicar debido a las diferencias de los sistemas constructivos. El trabajo de investigación tiene como objetivo producir un modelo de evaluación de sustentabilidad en la vivienda social, creado en base a las características y sistemas constructivos preponderantes en el contexto local. Se analizaron los modelos de sustentabilidad más conocidos a nivel mundial (BREEAM, CASBEE, LEED y VERDE) en las etapas del ciclo de vida del edificio que ellos evalúan y a partir de esta revisión se diseñó una metodología de evaluación para la vivienda social sustentable en el país; la cual se aplicó en un caso estudio local y arrojó como resultado la gran necesidad de reforzar las prácticas sustentables locales ya que el nivel de cumplimiento de los criterios definidos fue tan sólo un $52 \%$ de su totalidad.

Palablas-chaves: Modelo. Sustentabilidad. Vivienda. Ciclo de vida. Salvador Garcia Rodriguez
Tecnologico de Monterrey Monterrey - México

Miguel Davis Campoy Tecnologico de Monterrey Monterrey - México

Eva Campos Cantu Tecnologico de Monterrey Monterrey - México

Elizabeth Leyva Orihuela Tecnologico de Monterrey Monterrey - México

Recebido em 28/02/14

Aceito em 23/07/15

\begin{abstract}
Due to the strong environmental impact of the construction industry, there is great worldwide need for sustainable construction. The construction industry is the human activity that consumes the most natural resources, therefore systems have been developed to certify the level of sustainability and environmental impact of buildings. In Mexico no methodologies have been developed to assess the sustainability level of housing, and there is a widely acknowledged need to implement foreign models designed for another countries and other contexts, not feasible to apply due to the differences in building systems. The aim of this research project is to produce an assessment model for sustainability in social housing, shaped through the characteristics and main building systems of the local context. The most famous sustainability certifications worldwide were analyzed (BREEM, CASBEE, LEED AND $V E R D E)$ in the phases they evaluate of building life cycle, the result is the design of an assessment model for social housing; the methodology was applied in a local case study project and it was recognized as main result the need to strengthen the sustainable construction due to the poor fulfillment of criteria, that was $52 \%$ of the total value of the assessment.

Keywords: Model. Sustainability. Housing. Building lifecycle.
\end{abstract}




\section{Introducción}

El término Desarrollo Sostenible fue definido en 1987 en el Informe de la Comisión Mundial sobre el Medio Ambiente y el Desarrollo, se caracteriza por ser la forma de atender las necesidades presentes sin comprometer la capacidad de futuras generaciones para atender sus propias necesidades (BRUNDTLAND, 1987). La industria de la construcción es la principal actividad humana consumidora de recursos naturales por lo que las prácticas y tendencia de la construcción sustentable se están incrementando a nivel mundial y han ido modificando parámetros de construcción con el fin de producir proyectos que sean responsables con el medio ambiente.

El crecimiento demográfico mundial aumenta exponencialmente $y$ esto se ve reflejadomayormente en países en vía de desarrollo, demandando más uso de recursos y adaptación del territorio para habitar (MORALES, 2009). México, como país en vía de desarrollo enfrenta grandes retos que se desenvuelven a la par de su crecimiento, entre los retos principales está el dar vivienda digna a sus habitantes, hacer accesible vivienda que promueva bienestar, salud y calidad de vida de los usuarios. Se espera que para el 2030 México tenga más de 137 millones de habitantes, se necesitarán construir alrededor de 600,000 nuevas viviendas al año durante la próxima década,la tasa de crecimiento de proyección de hogares en México para el año 2030 será de más del 25\% (SISTEMA..., 2015).

Como dato complementario, debido la estructura de ingresos familiares en el País, la necesidad de abastecer vivienda de interés social es cada vez mayor, actualmente más del $60 \%$ de la demanda de vivienda se concentra en productos de vivienda social y económica (COMISIÓN..., 2015a), las cuales se adquieren principalmente mediante créditos. Una limitante para la adquisición de vivienda es el factor económico debido a la percepción de ingresos bajos:

Una enorme cantidad de personas no posee la oportunidad de recibir algún crédito por parte de una institución de seguridad social. Esto provoca un rezago importante en la capacidad de las personas de obtener una vivienda nueva institucional [...] (SÁNCHEZ CORRAL, 2012).

Una manera en que las comunidades han decidido resolver esta situación es mediante las "viviendas auto-construidas", lo cual representa un $60 \%$ de la edificación (SOCIEDAD..., 2012, 2014). El sector formal para la construcción de viviendas en los países de América Latina ha iniciado hasta hace no más de 3 décadas la construcción de casas, las cuales se plantean y diseñan sin tomar en cuenta las condiciones del terreno y los factores ambientales, trayendo graves consecuencias medioambientales, económicas y sociales al no considerar el impacto de los conceptos antes mencionados (Tabla 1) (SECRETARÍA..., 2014; COMISIÓN..., 2015a; COMISIÓN..., 2015b; CENTRO..., 2015). El factor climático como base para diseño y construcción de la vivienda no es tomado en cuenta por las entidades que planifican, diseñan y construyen viviendas, lo que da como resultado "viviendas formales" pero no adecuadas para el confort climático del usuario, por consiguiente, los usuarios de estas construcciones se ven obligados a buscar ese confort mediante la adquisición de equipamiento de aire acondicionado, generando gasto excesivo de energía eléctrica y más gasto económico.Por lo que es de gran importancia atender el problema al que se enfrenta el país a través del desarrollo de una solución sustentable factible para el contexto local.

En la Figura 1 se describe el ciclo de vida de una vivienda para mostrar las etapas en las que la aplicación de la sostenibilidad ayuda a obtener resultados más eficientes. En la etapa de diseño las acciones para guiar la construcción a un camino sustentable son más notorias porque estas acciones se ejecutan desde la planeación del proyecto.

Comparadas con el sistema constructivo tradicional, lasprácticas de la construcción sustentable conllevan grandes beneficios ambientales, económicos y socialespara todos los grupos involucrados: el país, gobierno, el desarrollador o constructor, usuario o propietario y la comunidad. Los modelos de sustentabilidad o certificación de edificios verdes son la evaluación de la creación de estructuras y la utilización de procesos que son responsables ambientalmente y eficientes en uso de recursos a través del ciclo de vida de los edificios y en todas sus etapas, desde la propuesta de diseño, la construcción, la operación, mantenimiento, la renovación, y la deconstrucción (MALDONADO RAMALLO, 2011). Se encargan de realizar el Análisis del Ciclo de Vida (ACV) para evaluar de la forma más objetiva posible, las cargas ambientales asociadas a un producto, proceso o actividad relacionado con el edificio identificando y cuantificando el uso de materia y energía y los vertidos al entorno para determinar el impacto que ese uso de recursos y esos vertidos producen en el medio ambiente, y para evaluar y llevar a la práctica estrategias de mejora ambiental.

8 Rodriguez, S. G.; Campoy, M. D.; Cantu, E. C.; Orihuela, E. L 
Tabla 1 - Impacto ambiental de la vivienda sin medidas de sustentabilidad

\begin{tabular}{|c|c|}
\hline \multicolumn{2}{|c|}{ IMPACTO AMBIENTAL } \\
\hline Concepto & Descripción \\
\hline $\begin{array}{l}\text { Consumo energético de } \\
\text { la vivienda }\end{array}$ & $\begin{array}{l}17.72 \% \text { del total del consumo de } \\
\text { energía eléctrica en México }\end{array}$ \\
\hline Emisión de gases & $\begin{array}{l}32 \% \text { del total de las emisiones de gas } \\
\text { que producen el efecto invernadero }\end{array}$ \\
\hline Residuos & $\begin{array}{l}36 \text { millones de toneladas anuales de } \\
\text { residuos urbanos en México }\end{array}$ \\
\hline Consumo de electricidad & $\begin{array}{l}26 \% \text { del consumo total de electricidad } \\
\text { en el país }\end{array}$ \\
\hline $\begin{array}{l}\text { Consumo de agua de } \\
\text { la vivienda }\end{array}$ & $\mid \begin{array}{l}14.1 \% \text { del agua potable en los } \\
\text { Estados Unidos }\end{array}$ \\
\hline $\mathrm{CO}_{2}$ industria de la construcción & $8.9 \%$ de las emisiones de $\mathrm{CO}_{2}$ \\
\hline $\mathrm{CO}_{2}$ sector & $7 \%$ \\
\hline
\end{tabular}

Fonte: Secretaría de Energía (2014), Comisión Nacional para el Uso Eficiente de la Energía (2015b) y Centro Mario Molina (2015).

Figura 1 - Ciclo de vida de una vivienda

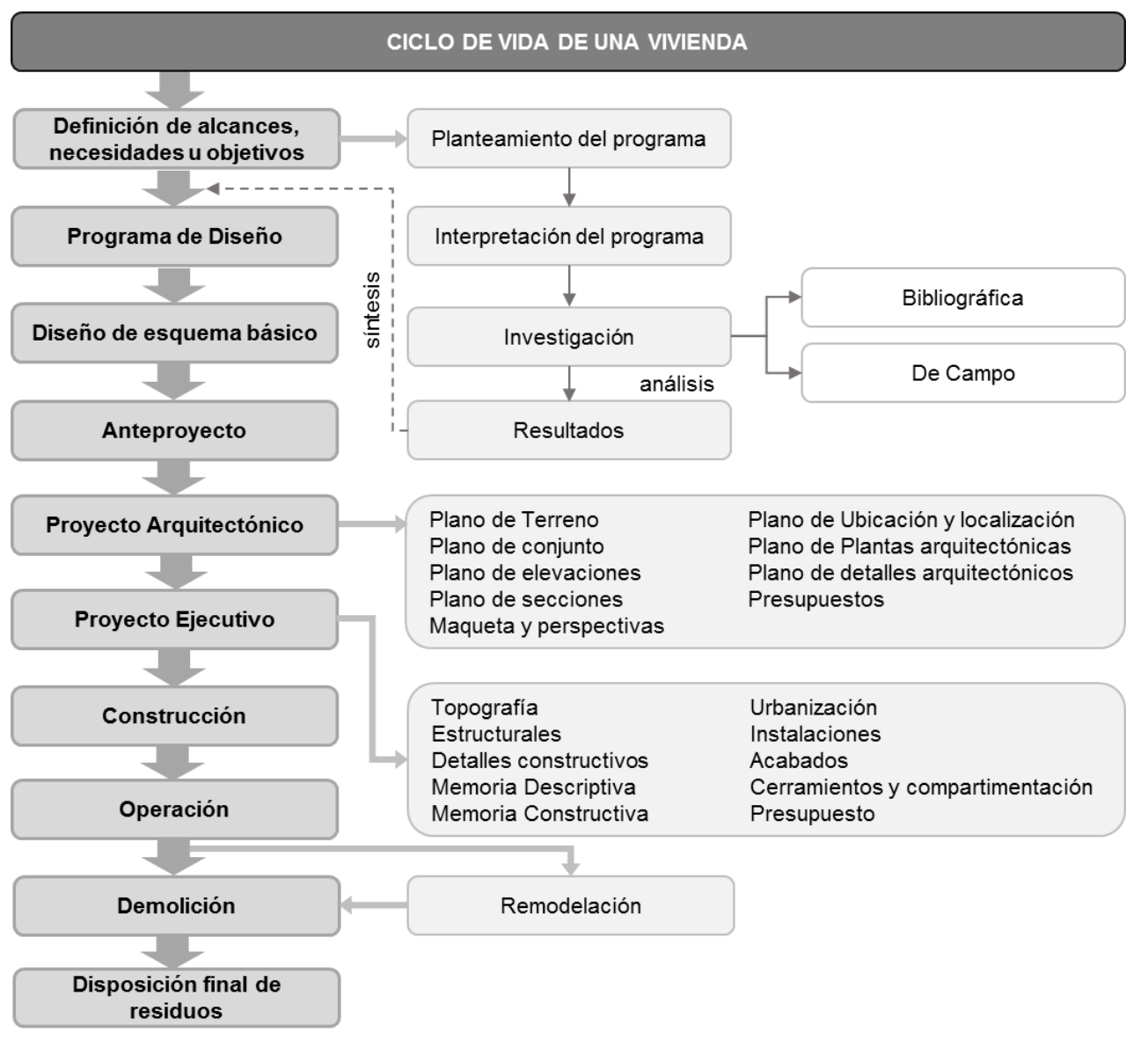

\section{Materiales y metodos}

Para satisfacer las necesidades de una vivienda sustentable en México es necesario un modelo de sostenibilidad que atienda las particularidades de ciclo de vida de la vivienda. Por lo anterior, se realizó una comparación entre los modelos de mayor aceptación y propagación en el mundo, analizando las etapas de impacto y conceptos que evalúan.

Los modelos de sostenibilidad que se utilizan para realizar el análisis se presentan a continuación en La Tabla 2. 
Tabla 2 - Descripción de sistemas de certificación

\begin{tabular}{|c|c|c|c|}
\hline \multicolumn{4}{|c|}{ SISTEMAS DE CERTIFICACIÓN } \\
\hline Nombre & $\begin{array}{l}\text { Lugar de } \\
\text { creación }\end{array}$ & Descripción & $\begin{array}{l}\text { Países de } \\
\text { aplicación }\end{array}$ \\
\hline $\begin{array}{l}\text { BREEAM: } \\
\text { Building Research } \\
\text { Establishment } \\
\text { Environmental Assessment } \\
\text { Method }\end{array}$ & Reino Unido & $\begin{array}{l}\text { Herramienta que utiliza un sistema simple y transparente de } \\
\text { puntuación asesorada por investigación basada en testeos reales, } \\
\text { influye en el diseño, construcción y mantenimiento de edificios } \\
\text { mediante un estándar técnico con garantías rigurosas de calidad y } \\
\text { certificación. }\end{array}$ & $\begin{array}{l}\text { Inglaterra, Holanda, } \\
\text { Noruega, España, } \\
\text { Suiza, Austria, } \\
\text { Alemania y más de } \\
\quad 50 \text { países }\end{array}$ \\
\hline $\begin{array}{l}\text { CASBEE: Comprehensive } \\
\text { Assessment System for } \\
\text { Building Environmental } \\
\text { Efficiency }\end{array}$ & Japón & $\begin{array}{l}\text { Evalúa y califica viviendas en términos de desempeño ambiental. } \\
\text { Busca proporcionar un buen ambiente de vida para utilizar las } \\
\text { viviendas por un largo tiempo y diseñar para ahorrar energía y } \\
\text { recursos para reducir la carga medioambiental y mejorar la calidad } \\
\text { de vida. }\end{array}$ & Japón \\
\hline Hipoteca Verde & México & $\begin{array}{l}\text { Programa creado para otorgar créditos hipotecarios para equipar las } \\
\text { viviendas con elementos ecológicos enlistados en una página de } \\
\text { productos autorizados. }\end{array}$ & México \\
\hline $\begin{array}{l}\text { LEED: } \\
\text { Leadership in Energy and } \\
\text { Environmental Design }\end{array}$ & Estados Unidos & $\begin{array}{l}\text { Es un criterio de medición que permitirá discernir si una edificación } \\
\text { es sustentable desde su diseño hasta su proceso constructivo, } \\
\text { basándose en los más altos estándares de desempeño en } \\
\text { eficiencia energética y bajo impacto al medio ambiente. }\end{array}$ & $\begin{array}{l}\text { Estados Unidos, } \\
\text { Latinoamérica } \\
\text { excepto Cuba, más } \\
\text { de } 110 \text { países }\end{array}$ \\
\hline $\begin{array}{l}\text { VERDE: } \\
\text { Valoración de Eficiencia de } \\
\text { Referencia de Edificios }\end{array}$ & España & $\begin{array}{l}\text { Evalúa mediante la comparación del edificio proyectado con un } \\
\text { edificio de referencia estándar realizado con las exigencias } \\
\text { mínimas fijadas por las normas y la práctica común. }\end{array}$ & $\begin{array}{l}\text { España, Australia, } \\
\text { Sudáfrica }\end{array}$ \\
\hline
\end{tabular}

Los modelos descritos en la tabla anterior nacen del punto de vista de los gobiernos y asociaciones no gubernamentales que se interesaron en mejorar la forma en que se construyen los edificios, pero cada uno es particular a los procedimientos constructivos más utilizados en las naciones donde se crearon. Como ejemplo, el modelo CASBEE solamente se ha podido implementar con éxito dentro de Japón (más de 350 edificios) hasta el año 2014 en Tianjin, China, se certificó el primer edificio fuera de Japón (COMPREHENSIVE..., 2014), mientras que modelos como LEED, BREEAM o VERDE, son utilizados en más países en donde se han tratado de adaptar a los sistemas constructivos del país, pero suelen ser más rígidos para su implantación.

Para comparar los modelos de sustentabilidad se obtuvo el manual para acreditar una vivienda de sus respectivas páginas de internet, se tomaron en cuenta los temas que evalúan y la ponderación general que asignan a cada rubro (Tabla 3 ).

Caso aparte se encuentra el modelo de CASBEE (COMPREHENSIVE..., 2014), su sistema de evaluación utiliza otra metodología que se presenta en la Tabla 4; y el modelo de Hipoteca Verde de México, ya que este modelo sólo permite agregar un crédito adicional a la adquisición de vivienda del usuario según el salario que percibe, para comprar equipamientos asignados como eco tecnologías que ayudarán a reducir gastos de operación en los servicios primarios (agua, electricidad) (INFONAVIT, 2014). En la Tabla 5 se listan los ahorros mínimos mensuales producidos por las eco tecnologías en base a la percepción salarial mensual del derechohabiente.

En México existe una gran necesidad de brindar al usuario una oferta de vivienda integral sustentable, lo que dio pie al diseño de un modelo para evaluar la sustentabilidad en la vivienda sin necesidad de adaptarse a los estándares que proponen los modelos anteriormente analizados. La propuesta de evaluación fue elaborada mediante el análisis de los modelos sustentables más replicados en el mundo buscando su adaptación a los sistemas constructivos locales. A través de la literatura revisada se estableció un método para el diseño del modelo de evaluación. Se definieron las principales variables a evaluar para la definición de clasificaciones y conceptos a incluir; para éstos se consideró todo el ciclo de vida del proyecto, desde la concepción y diseño hasta la operación y demolición de la edificación, mediante herramientas y/o técnicas de diseño bioclimático, flexibilidad, constructabilidad, gestión de la construcción, gestión de residuos y en la operación de la edificación. Se integró un panel de expertos conformado por colaboradores de la empresa e institución educativa participantes y a través de este panel se realizó un proceso de clasificación y depuración de conceptos, posteriormente se procedió a definir el sistema para evaluar y ponderar las clasificaciones y conceptos definitivos. 
Tabla 3 - Análisis comparativo de los modelos de sostenibilidad

\begin{tabular}{|l|c|c|c|}
\hline \multicolumn{4}{|c|}{ TABLA DE EVALUACIÓN } \\
\hline \multirow{2}{*}{ Áreas que evalúan } & \multicolumn{3}{c|}{ Sistemas a evaluar } \\
\cline { 2 - 4 } & BREEAM & LEED & VERDE \\
\hline Gestión & 10 & 42 & 2.8 \\
\hline Calidad Ambiental Interior & 14 & 17 & 13.8 \\
\hline Energía & 22 & 37 & 42.7 \\
\hline Transporte & 8 & 0 & 0 \\
\hline Agua & 12 & 11 & 0 \\
\hline Materiales & 14 & 14 & 33 \\
\hline Uso de la Tierra y Ecología & 10 & 21 & 7.5 \\
\hline Emisiones & 10 & 0 & 0 \\
\hline Innovación & 0 & 64 & 5 \\
\hline Total & $\mathbf{1 0 0}$ & $\mathbf{1 1 0}$ & $\mathbf{1 0 5}$ \\
\hline
\end{tabular}

Tabla 4 - Análisis comparativo del modelo CASBEE

\begin{tabular}{|c|c|c|c|}
\hline \multicolumn{4}{|c|}{ EVALUACIÓN CASBEE } \\
\hline Concepto & Subconcepto & Valor & $\begin{array}{l}\text { Valor } \\
\text { total }\end{array}$ \\
\hline \multirow{3}{*}{$\begin{array}{l}\text { Calidad del } \\
\text { medioambiente }\end{array}$} & Interior del edificio confortable y saludable & 4.8 & \multirow{3}{*}{4.4} \\
\hline & Aseguramiento de larga vida del producto & 3.8 & \\
\hline & Crear un entorno ambiental amigable & 4.6 & \\
\hline \multirow{3}{*}{$\begin{array}{l}\text { Reducción de } \\
\text { carga ambiental }\end{array}$} & Conservación de la energía y agua & 4.3 & \multirow{3}{*}{3.9} \\
\hline & Gestión de recursos y reducción de residuos & 3.2 & \\
\hline & $\begin{array}{l}\text { Afectación al medio ambiente global, local y } \\
\text { alrededores }\end{array}$ & 4.8 & \\
\hline
\end{tabular}

Fonte: Comprehensive Assessment System for Built Environment Efficiency (2007).

Tabla 5 - Rangos de ahorro mensual (Pesos Mexicanos, 1 dólar americano $=16$ pesos mexicanos) con el uso de eco-tecnologías

\begin{tabular}{|c|c|c|c|}
\hline \multicolumn{4}{|c|}{ HIPOTECA VERDE } \\
\hline \multicolumn{3}{|c|}{ Ingreso (salario integrado mensual) } & $\begin{array}{l}\text { Ahorro } \\
\text { mínimo }\end{array}$ \\
\hline \multirow{5}{*}{ Rangos } & $\$ 2,045.62$ & $\$ 3,272.98$ & $\$ 100.00$ \\
\hline & $\$ 3,272.99$ & $\$ 88,182.45$ & $\$ 215.00$ \\
\hline & $\$ 8,182.46$ & $\$ 14,319.30$ & $\$ 250.00$ \\
\hline & $\$ 14,319.31$ & $\$ 22,501.77$ & $\$ 290.00$ \\
\hline & $\$ 22,501.78$ & en adelante & $\$ 400.00$ \\
\hline
\end{tabular}

Fonte: Infonavit (2014).

La línea de investigación y objetivos primordiales fueron los siguientes:

(a) revisión de Modelos y Metodologías existentes para la evaluación de la sustentabilidad en las edificaciones; (b) realización de las características, fortalezas y debilidades de cada uno de los modelos y metodologías revisados;

(c) realización de una metodología Integral de Evaluación Sostenible de un Prototipo de Vivienda Social; y 
(d) aplicación de la metodología en Proyecto Real y Retroalimentación del mismo.

\section{Resultados}

Los objetivos anteriores dieron como resultado una metodología lo suficientemente flexible para adecuarse a las diversas condiciones que presenta el sector inmobiliario en México. En la Figura 2 se presenta ladescripción conceptual del modelo.

La Figura 3 muestra un fragmento de la interface, ejemplo de formato y estructura de elementos que integran de la metodología resultante después del análisis de los modelos sustentables más representativos en el mundo y con la inclusión de las mejoras que se consideraron pertinentes para su fácil adaptación al contexto local.

A continuación se describen los elementos que integran el formato de la metodología:

(a) clasificación: es la agrupación general de los conceptos que integran la metodología de evaluación (Tabla 6). La clasificación se compone de6 importantes grupos: diseño bioclimático, Ecotecnias, Soluciones técnico - constructivas, Gestión de residuos, Calidad y Seguridad; y Potencia de desarrollo;

(b) ponderación: indica el valor de la respectiva "Clasificación" expresado en términos de "Porcentaje". La ponderación se estructura en 3 grupos principales y se define en base al nivel de impacto de la clasificación en el producto final, asignando un $45 \%$ para las clasificaciones de Diseño, un $35 \%$ para las de Construcción y un $20 \%$ para las de Uso (Tabla 6);

(c) concepto: representan el subgrupo que integra los parámetros que poseen las mismas cualidades. La metodología de evaluación se compone de 14 conceptos, ilustrados en la Tabla 6; catalogados cada uno dentro de alguna de las 6 clasificaciones existentes. Los conceptos son Constructabilidad, Diseño, Energías Alternativas, Materiales,
Emisiones a la atmósfera, Emisiones de polvo y partículas, Afectación al suelo, Consumo de agua, Consumo de materiales, Gestión de residuos, Calidad en el proceso, Calidad en el producto final, Seguridad y Estabilidad del usuário;

(d) clave: representa el dígito identificador de los criterios/parámetros existentes en la metodología de evaluación;

(e) parámetro: son la parte medular de la metodología de evaluación, son los mecanismos que permitirán evaluar el grado de sustentabilidad de un determinado proyecto. La obtención de estos parámetros, se realizó con reuniones con el equipo de Cemex vivienda, Cemex Energia, Cemex Innovación, Cemex Suiza y Tecnológico de Monterrey, en donde cada parte propuso parámetros y estos fueron depurados con reuniones en conjunto.Se han realizado mediante un mecanismo de preguntas, en el cual el evaluador examinará si la determinada vivienda cumple de manera satisfactoria cada uno de los diversos parâmetros;

(f) esfera: representa la etapa de clasificación en la cual se involucra el parámetro al momento de la evaluación. En total son 6 que son: Diseño bioclimático, Ecotecnias, Soluciones técnico constructivas, Gestión de residuos, Calidad y Seguridad; y Potencia de desarrollo;

(g) tolerancia: indica las pautas que debe cumplir la obra en evaluación para que se asigne la puntuación del parámetro sobre el cual se está evaluando;

(h) rango de cumplimiento: determina si el proyecto que se está evaluando cumple con el respectivo parámetro sobre el cual se está evaluando o no; y

(i) ponderación de parâmetros: representa el valor del parámetro evaluador dentro de la metodología de evaluación expresado en "Puntos". 
Figura 2 - Descripción conceptual del modelo

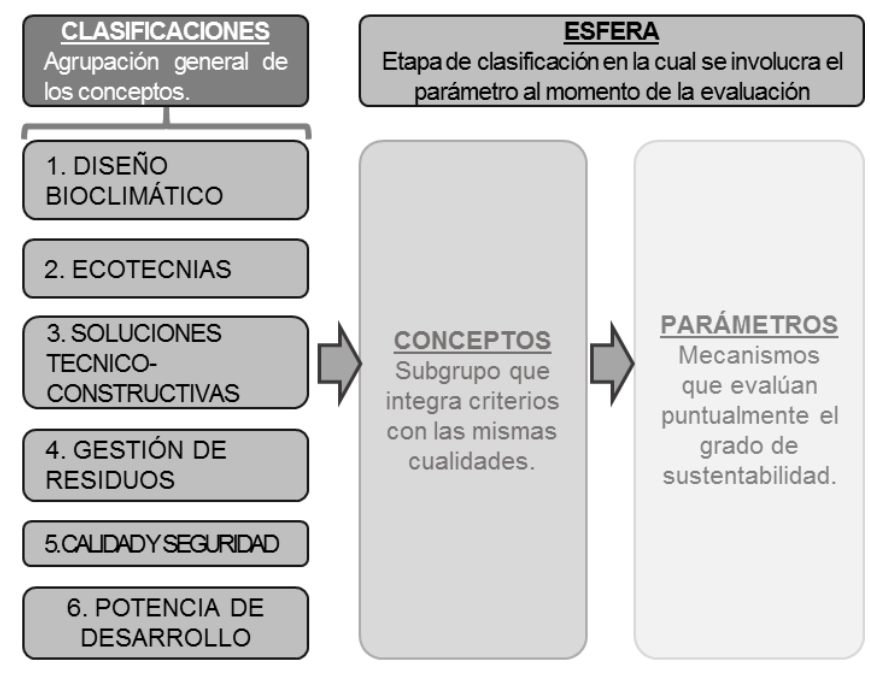

Figura 3 - Ejemplo del formato de la metodologia

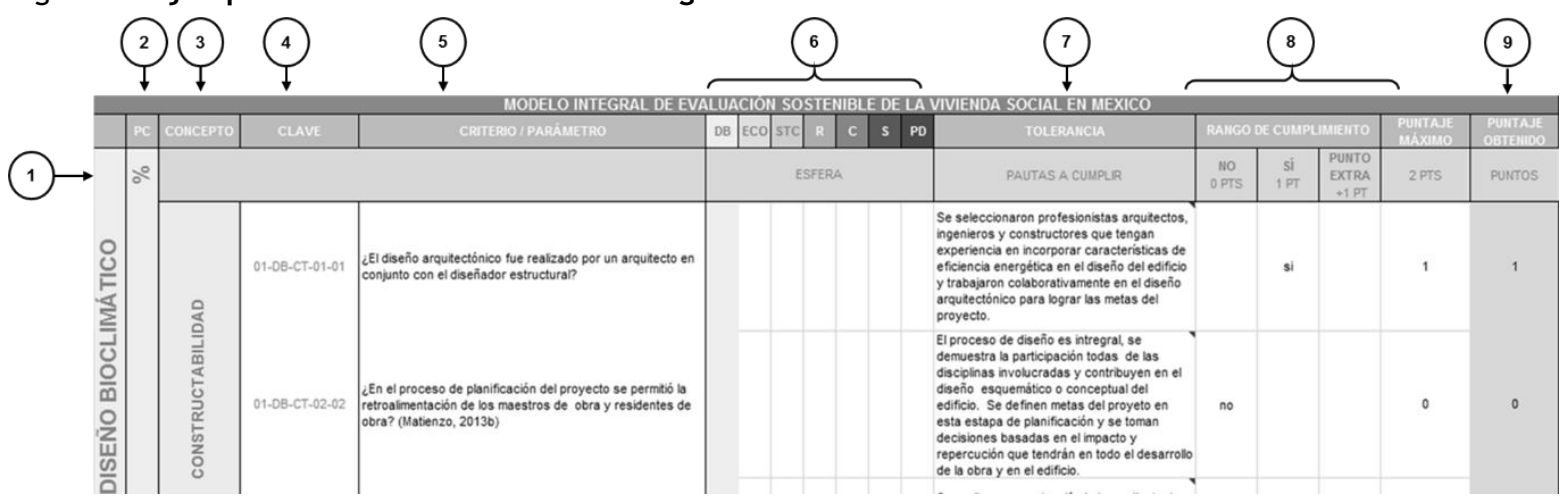

Tabla 6 - Resumen de clasificaciones y conceptos que analiza la metodología

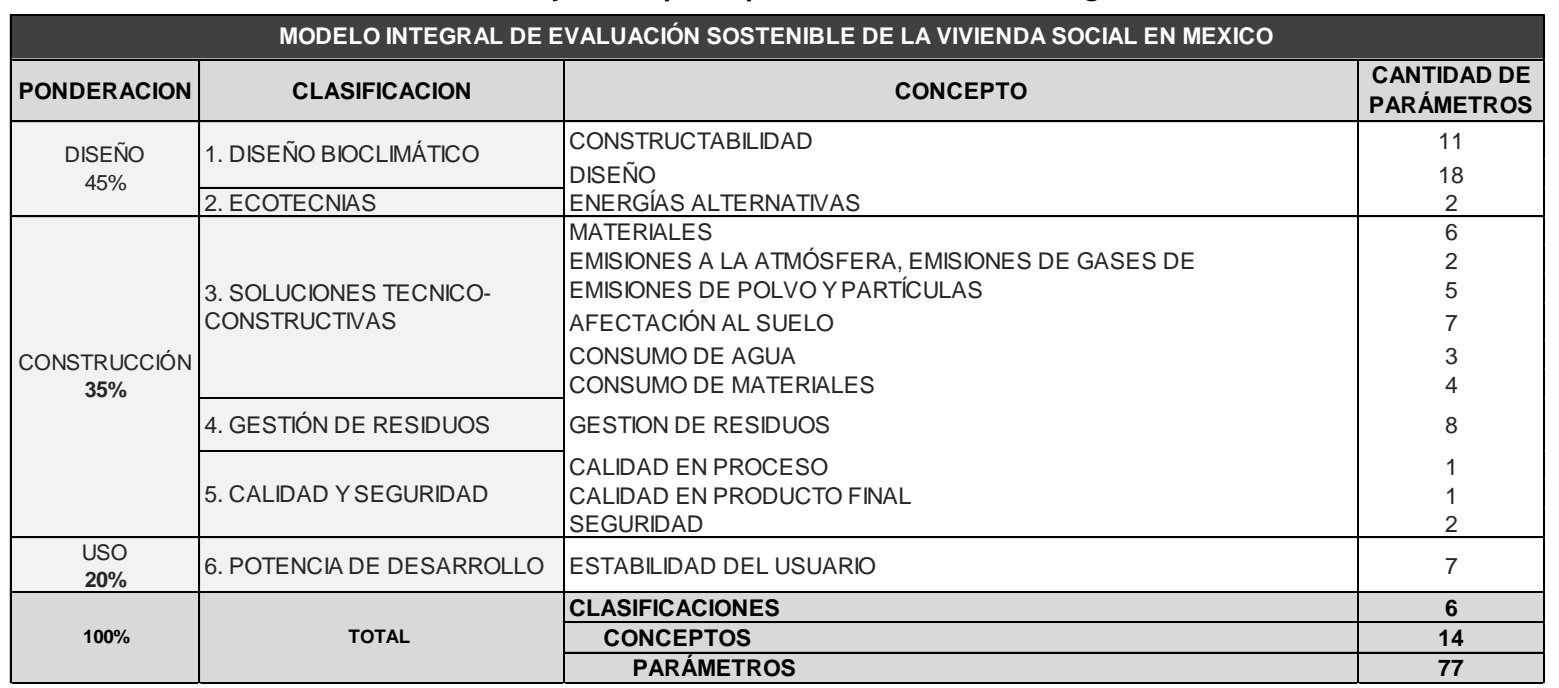




\section{Guía práctica de utilización}

La metodología de evaluación se encuentra dividida en 6 grandes rubros o "Clasificaciones" que son Diseño bioclimático, Ecotecnias, Soluciones técnico - constructivas, Gestión de residuos, Calidad y Seguridad; y Potencia de desarrollo (Tabla 6). Las "clasificaciones" a su vez se encuentran compuestas por subgrupos conocidos como "Conceptos" que suman un total de 14, la función principal de los "Conceptos" es agrupar los "Parámetros" que posean las mismas características o cualidades.Las casillas de "Clasificación", "Conceptos" y "Esferas" son elementos que sólo funcionan para agrupar los "Parámetros" los cuales son la parte medular de la metodología de evaluación.

La forma en que se realiza la evaluación de un proyecto es la Figura 4.

La forma en que se utiliza la guía es la siguiente:

(a) una vez que se ha definido la obra a evaluar, habrá que definir sobre cuál rubro se calificará primero. No existe un orden específico para la calificación, pero se recomienda hacerlo en el orden estructurado en la guia;

(b) se identifica el "Parámetro" a evaluar, se analizan las "tolerancias" que deben tomarse en cuenta para afirmar que el proyecto cumple o no con este"parámetro";

(c) los "parámetros" están redactados en forma de preguntas, a través de las cuales se define si el proyecto cumple o no con los requerimientos especificados en el "nivel de tolerancia" para dicho "parámetro";

(d) si el proyecto cumple con los requerimientos mínimos que el "Nivel de Tolerancia" indica, se procede a marcar la casilla "Sí", en caso contrario, marcar "No",

(e) al marcar la casilla "Sí" se asigna automáticamente 1 punto, la casilla "No" asigna 0 puntos; y

(f) la casilla "Punto extra" asigna un punto adicional al "parámetro" si el proyecto cumple con los requerimientos mínimos especificados y los sobrepasa alcanzando niveles ideales de cumplimiento superiores al "nivel de tolerancia" del "parámetro", por lo que se le asigna un punto extra marcando la casilla "si" en la columna "punto extra".

\section{Aplicación en caso estudio}

Con el fin de conocer las fortalezas y debilidades del modelo de evaluación creado y canalizar vías de mejora, se procedió a su aplicación en un proyecto de vivienda de Interés social ubicado en la Zona Metropolitana de Monterrey, el proyecto se realiza con los estándares constructivos preponderantes en la localidad, los cuales son una construcción tradicional a base de block o de ladrillo, con losa de concreto o vigueta y bovedilla y representa la oferta típica de vivienda de interés social. Los resultados obtenidos se ilustran en la Tabla 7 .

\section{Figura 4 - Ejemplo evaluación de un proyecto}

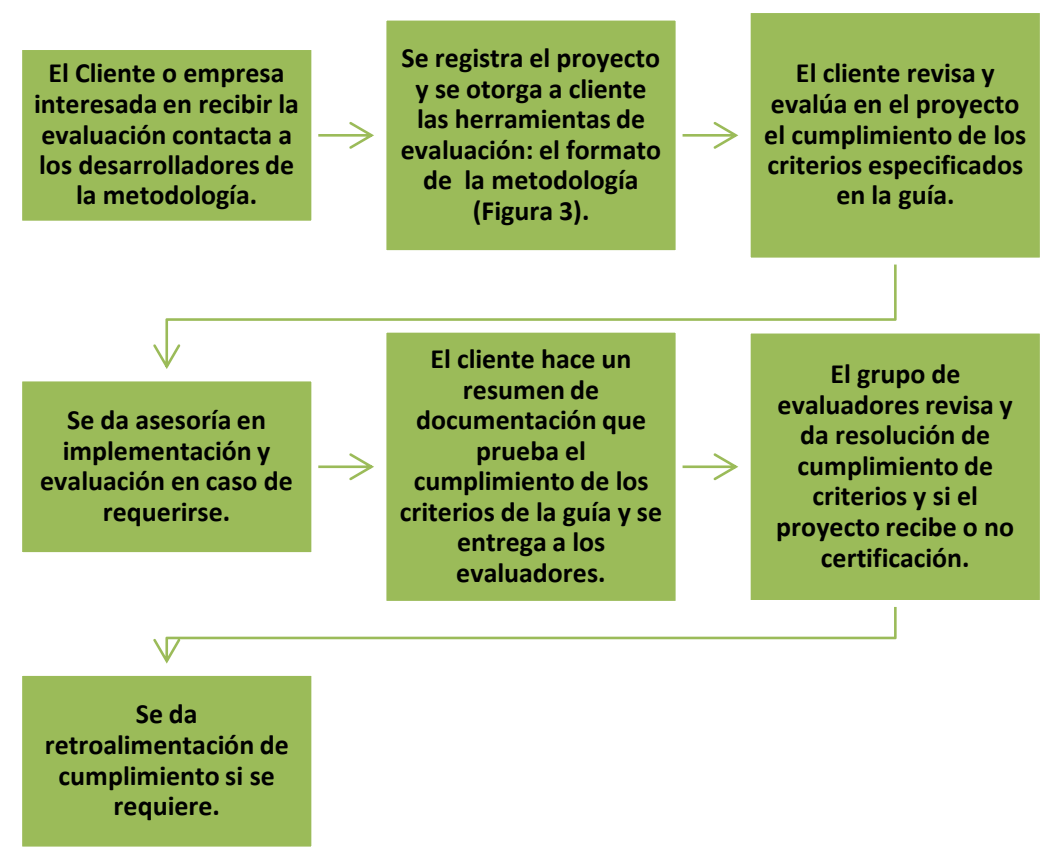

14 Rodriguez, S. G.; Campoy, M. D.; Cantu, E. C.; Orihuela, E. L 
Tabla 7 - Resultados de aplicación

\begin{tabular}{|c|c|c|c|c|}
\hline \multicolumn{5}{|c|}{$\begin{array}{l}\text { MODELO INTEGRAL DE EVALUACIÓN SOSTENIBLE } \\
\text { DE LA VIVIENDA SOCIAL EN MEXICO } \\
\text { RESUMEN DE EVALUACIÓN }\end{array}$} \\
\hline CLASIFICACION & $\begin{array}{c}\text { TOTAL } \\
\text { PARÁMETROS }\end{array}$ & $\begin{array}{l}\text { PARÁMETROS } \\
\text { CUMPLIDOS }\end{array}$ & PONDERACION & $\begin{array}{c}\text { PUNTAJE } \\
\text { OBTENIDO }\end{array}$ \\
\hline \multirow{2}{*}{ 1. DISEÑO BIOCLIMÁTICO } & 11 & 9 & \multirow{3}{*}{$\begin{array}{l}\text { DISEÑO } \\
45 \%\end{array}$} & \multirow{3}{*}{$24.68 \%$} \\
\hline & 18 & 6 & & \\
\hline 2. ECOTECNIAS & 2 & 2 & & \\
\hline \multirow{6}{*}{$\begin{array}{l}\text { 3. SOLUCIONES TECNICO- } \\
\text { CONSTRUCTIVAS }\end{array}$} & 6 & 2 & \multirow{10}{*}{$\begin{array}{c}\text { CONSTRUCCIÓN } \\
35 \%\end{array}$} & \multirow{10}{*}{$16.15 \%$} \\
\hline & 2 & 2 & & \\
\hline & 5 & 1 & & \\
\hline & 7 & 4 & & \\
\hline & 3 & 2 & & \\
\hline & 4 & 2 & & \\
\hline 4. GESTIÓN DE RESIDUOS & 8 & 3 & & \\
\hline \multirow{3}{*}{ 5. CALIDAD Y SEGURIDAD } & 1 & 1 & & \\
\hline & 1 & 0 & & \\
\hline & 2 & 1 & & \\
\hline $\begin{array}{l}\text { 6. POTENCIA DE } \\
\text { DESARROLLO }\end{array}$ & 7 & 4 & $\begin{array}{l}\text { USO } \\
20 \% \\
\end{array}$ & $11.43 \%$ \\
\hline TOTAL & 77 & 39 & $100 \%$ & $52.25 \%$ \\
\hline
\end{tabular}

La aplicación de la metodología de evaluación en un proyecto típico de vivienda social en la localidad permite reconocer lo siguiente:

(a) el porcentaje de cumplimiento de estándares de sustentabilidad en la oferta actual de vivienda de interés social en la localidad es bajo ya que apenas supera el 50\% de los "parámetros" totales que la metodología evalúa; y

(b) la "clasificación" 1. Diseño bioclimático es la de mayor peso y la de mayor cantidad de "parámetros" a evaluar y en este caso en específico sólo el $24.68 \%$ de la ponderación es de $45 \%$.

\section{Conclusiones}

Es un hecho que la preocupación por el medio ambiente ha dejado de ser un concepto de moda para pasar a ser una necesidad explicita en todas las industrias donde se desempeña el ser humano. La industria de la construcción ha adoptado el término de sustentabilidad en amplias áreas desde procesos de fabricación de los materiales, así como en el ciclo de vida de los edificios. Si bien, los modelos de sustentabilidad creados por las organizaciones no gubernamentales han tenido un impacto positivo dentro de la construcción en los últimos 20 años, aún son muy estrictos para su aplicación en todas las naciones por la falta de uniformidad de los procesos constructivos.

Son pocos los modelos de sustentabilidad que se adaptan a las necesidades de los sistemas constructivos en México, por ser diseñados por países con otros tipos de sistemas constructivos. Es necesario que haya iniciativa gubernamental en la promoción y colaboración con las asociaciones civiles el estudio de los procedimientos para diseñar modelos que evalúen la sustentabilidad, ajustados a sus necesidades locales.

Esta investigación arrojó una metodología diseñada mediante instituciones educativas e industria privada que lograron aportar una herramienta práctica de ejecutar en México por el conocimiento del sistema constructivo en la región que se diseñó. También se pudo realizar una implementación sin algún problema en su implementación lo que nos sugiere que para el construcción o desarrollador inombiliario no representa un problema la implementación. Como futuras investigaciones, se sugiere comparar la evaluación de esta herramienta aplicada en un edificio con los demás modelos de sustentabilidad para mostrar la eficiencia de su metodología y encontrar las áreas de oportunidad que se pueden mejorar para sus futuras versiones.

\section{Bibliografía}

BRUNDTLAND, G. H. Our Common Future.

World Commission on Environment and Development, 1987.

CENTRO MARIO MOLINA. Evaluación de la Sustentabilidad de la Vivienda en México.

Disponíble en: <http://centromariomolina.org/wpcontent/uploads/2012/09/14.-

Evaluaci\%C3\%B3nSustetabilidadViviendaM\%C3

\%A9xico_fin.pdf>. Acceso en: 08 set. 2015. 
COMISIÓN NACIONAL DE VIVIENDA. NAMA de Vivienda Existente 2013. Disponíble em:

<http://www.conavi.gob.mx/viviendasustentable>. Acceso em: 7 set. 2015b.

COMISIÓN NACIONAL DE VIVIENDA. Tipología de Vivienda. Abr. 2015. Disponíble em: <http://www.conavi.gob.mx/tipologia-devivienda>. Acceso em: 7 set. 2015 c.

COMISIÓN NACIONAL PARA EL USO EFICIENTE DE LA ENERGÍA. Estrategia Nacional Para la Vivienda Sustentable. Disponíble em: <http://www.conuee.gob.mx/pdfsvivienda/FIDEA Embbritanicaestrategianacionalviviendasustentable f.pdf>. Acceso em: 7 set. 2015b.

COMPREHENSIVE ASSESSMENT SYSTEM FOR BUILT ENVIRONMENT EFFICIENCY. CASBEE for Home (Detached House). 2007. Technical Manual. Disponíble en: <http://www.ibec.or.jp/CASBEE/english/downloa d/CASBEE-H(DH)e_2007manual.pdf>. Acceso em: 7 set. 2015 .

COMPREHENSIVE ASSESSMENT SYSTEM FOR BUILT ENVIRONMENT EFFICIENCY. Estadísticas CASBEE. 2014. Disponíble em: <http://www.ibec.or.jp/CASBEE/english/statistics. htm>. Acceso em: 7 set. 2015.

INFONAVIT. Hipoteca Verde. México, 2014. Disponíble em:

<http://portal.infonavit.org.mx/wps/wcm/connect/i nfonavit/trabajadores/saber+para+decidir/cuido_m i_casa/ahorro+y+cuido+el+medio+ambiente/ahorr $\mathrm{o}+\mathrm{y}+$ cuido+el+medio+ambiente>. Acceso em: 7 set. 2015.

MALDONADO RAMALLO, M. Estudio Comparativo de Certificaciones "Green Building” en Edificios, Para la Elaboración de Un Modelo Inicial Para América del Sur. Maestría - Universitat Politècnica de Catalunya, Barcelona, España. 2011.
MORALES, I. La Importancia del Desarrollo Sostenible. Economía + Desarrollo. 2009. Disponível em:

<https://economiaydesarrollo.wordpress.com/2009 /01/31/desarrollo-sostenible/>. Acesso em: 08 set. 2015.

SÁNCHEZ CORRAL, J. La Vivienda Social en

México: pasado, presente y futuro? México, 2012. Disponíble en:

<http://www.jsa.com.mx/documentos/publiaciones _jsa/libro\%20vivienda\%20social.pdf $>$. Acceso en : 7 set. 2015.

SECRETARÍA DE ENERGÍA. Balance Nacional de Energía 2013. México: SENER, 2014.

Disponíble em:

<http://www.sener.gob.mx/res/PE_y_DT/pub/2013

/Balance_2013.pdf $>$. Acceso em: 7 set. 2015.

SISTEMA NACIONAL DE INFORMACIÓN E INDICADORES DE VIVIENDA. Proyección de Hogares de México y las Entidades Federativas 2010-2030 de CONAPO. Abr. 2015a. Disponíble en: <http://www.conavi.gob.mx/introduccion>. Acceso em: 7 set. 2015.

SOCIEDAD HIPOTECARIA FEDERAL. ¿Por Qué Autoproducción de Vivienda Asistida? 2012. Disponíble em:

<http://www.conorevi.org.mx/pdf/Evento29y30ag osto/AVA.pdf>. Acceso em: 7 set. 2015.

SOCIEDAD HIPOTECARIA FEDERAL. Esquemas de Financiamiento: servicios, calidad y espacio en la vivienda. 2014. Disponíble em: <http://www.sedesol.gob.mx/work/models/SEDES OL/PDF/ForoPronades/05_Vivienda_03.pdf $>$. Acceso em: 7 set. d2015.

\section{Agradecimientos}

A la institución ITESMy a la empresa CEMEX por el apoyo y patrocinio para la realización de este proyecto de investigación. 


\section{Salvador Garcia Rodriguez}

Departamento de Ingenieria civil | Tecnologico de Monterrey | Av. Eugenio Garza Sada, 2501 | CP 64849 | Monterrey - México | Tel.: +52 (81) 8358-2000 | E-mail: migueldavis@itesm.mx

\section{Miguel Davis Campoy}

Departamento de Ingenieria civil | Tecnologico de Monterrey | E-mail: migueldavis@itesm.mx

\section{Eva Campos Cantu}

Departamento de Ingenieria civil | Tecnologico de Monterrey | E-mail: eba.campos@hotmail.com

Elizabeth Leyva Orihuela

Departamento de Ingenieria civil | Tecnologico de Monterrey | E-mail: eli_leyvaori@hotmail.com

\section{Revista Ambiente Construído}

Associação Nacional de Tecnologia do Ambiente Construído

Av. Osvaldo Aranha, $99-3^{\circ}$ andar, Centro

Porto Alegre - RS - Brasil

CEP $90035-190$

Telefone: +55 (51) 3308-4084

Fax: +55 (51) 3308-4054

www.seer.ufrgs.br/ambienteconstruido

E-mail: ambienteconstruido@ufrgs.br 